\title{
Antidepressant use in adults with intellectual disability
}

\author{
Prem R. Rai, ${ }^{1}$ Mike Kerr ${ }^{2}$
}

The Psychiatrist (2010), 34, 123-126, doi: 10.1192/pb.bp.108.023325

${ }^{1}$ Aneurin Bevan Health Board, Torfaen, Wales; ${ }^{2}$ Welsh Centre for Learning Disability, Cardiff

Correspondence to Prem R. Rai (premrai50@hotmail.com)

\begin{abstract}
Aims and method To study the use of antidepressants in adults with intellectual disability, focusing on medication type, indication, retention and clinical outcome. Case notes of all service users in a learning disability service were hand-searched to identify antidepressant usage, and those who had been treated with antidepressants and in whom at least 1 year of follow-up was possible were included in the study.
\end{abstract}

Results A total of 241 treatment episodes were identified. The rates of positive outcome in terms of clinical improvement at 6 weeks, 6 months and 12 months were $49.4 \%, 48.1 \%$ and $49 \%$ respectively, and only 29 (12\%) episodes of side-effects had been noted.

Clinical implications Antidepressants are commonly prescribed in adults with intellectual disability. Approximately half did well in terms of clinical improvement.

Declaration of interest None.
Mental ill-health is common in adults with intellectual disability. A recent study has found point prevalence rates for affective disorder and anxiety disorder at $6.6 \%$ and $3.8 \%$ respectively, and prevalence rates for problem behaviour and obsessive-compulsive disorder at $22.5 \%$ and $0.7 \%$ respectively. ${ }^{1}$ These point prevalence rates are higher than those observed in the general population. ${ }^{2}$

The research on the efficacy of antidepressants in people with intellectual disability comprises mainly case reports and small open studies. In an open trial of fluoxetine involving six individuals with intellectual disability who had depression, Howland found 'positive result' in all. ${ }^{3}$ Langee \& Conlon analysed the case notes of 149 adult in-patients with severe intellectual disability and concluded that those with depressive, psychotic and behavioural problems responded positively to tricyclics and tetracyclics. ${ }^{4}$ An open study of the use of citalopram in 20 patients with intellectual disability and depression found significant improvement in 12 of them. ${ }^{5}$ Bhaumik et al studied 122 case notes and found that adults with intellectual disability responded to fluoxetine and paroxetine in depression. ${ }^{6}$

In addition, further non-randomised or controlled studies have been reported in problem behaviours including self-injurious and perseverative behaviours. ${ }^{7,8}$ One review looked at all the published studies from 2003 to 2004 in intellectual disability and depression and found no study on the treatment. ${ }^{9}$

In the context of this paucity of evidence, we felt it important to explore the usage of antidepressants in a large National Health Service (NHS) trust, focusing on usage, retention, safety and clinical outcomes.

\section{Method}

We retrospectively analysed all the case notes available on adults with intellectual disability receiving input from Abertawe Bro Morgannwg University Health Board Learning Disability Directorate in south Wales. The service covers a population of over 600000 , with six full-time consultants in learning disability psychiatry. We included those service users who had received a new treatment with antidepressants within the past 5 years and excluded those for whom 12-month follow-up was not possible, as well as those who were receiving antidepressants for physical illnesses. The case notes were examined for the following variables: gender, age, housing, diagnosis, antidepressant used, comorbidity, co-prescription, level of intellectual disability and past history of depression. Treatment outcome was assessed in terms of clinical improvement as recorded in notes, retention of antidepressant, and sideeffects at or around 6 weeks, 6 months and 12 months after the initiation of treatment. Clinical improvement was noted as no change, significant improvement, back to normal and worse. Consultants involved were contacted to clarify issues when required.

We used SPSS 14.0 for Windows to analyse data for the frequencies and percentages of the variables. As the local research and ethics committee classified the project as an audit, no consent was required.

\section{Results}

\section{Sample characteristics}

The total number of case notes studied was 221, of which 103 were for males (mean age 41 years, range 19-77) and 118 
for females (mean age 43.5 years, range 19-72). As regards the severity of intellectual disability, this was mild in $43 \%$ of the sample, moderate in $26.2 \%$, severe in $16.3 \%$, profound in $2.3 \%$ and borderline intellectual disability in $2.7 \%$; in $9.5 \%$ of the sample, the level of intellectual disability was not classified.

The underlying cause of intellectual disability was not known in $77 \%$ of the cases. The most common known cause was Down syndrome (10.8\%), with the following other main causes: cerebral palsy (3.1\%), Angelman syndrome (1.8\%), fragile-X syndrome (1.3\%), Prader-Willi syndrome (0.9\%), birth injury (0.9\%) and childhood encephalitis (0.9\%).

The majority of service users were either living in supported residential homes $(51.8 \%, n=114)$ or with their families $(35.7 \%, n=79)$. Of the remaining sample, seven were in-patients $(3.2 \%)$, five $(2.2 \%)$ lived independently and others were in adult or other placements.

Case-note analysis revealed a total of 241 treatment episodes. Antidepressants were used for depression in 147 episodes (61.3\%), for generalised anxiety disorder in 24 episodes (9.9\%) and for obsessive-compulsive disorder in 22 episodes (9.1\%). The full range of indicators for the use of antidepressants is presented in Table 1.

Out of 221 service users, $41(24 \%)$ had a history of epilepsy; 21 had active epilepsy and 20 had not had seizures for 1 year or longer. Of those who had active epilepsy, twothirds were having fewer than 12 seizures per year and a third were experiencing more than 12 seizures per year. Challenging behaviour was present in 96 (43.4\%) of the sample.

\begin{tabular}{|c|c|c|}
\hline Diagnosis & $\begin{array}{c}\text { Treatment } \\
\text { episodes, }{ }^{a} \\
n\end{array}$ & $\begin{array}{c}\text { Total } \\
\text { episodes, } \\
\%\end{array}$ \\
\hline Depression & 147 & 61.3 \\
\hline Generalised anxiety disorder & 24 & 9.9 \\
\hline Obsessive-compulsive disorder & 22 & 9.1 \\
\hline Mixed anxiety and depressive disorder & 11 & 4.6 \\
\hline Bipolar disorder & 9 & 3.8 \\
\hline Behaviour disorder & 8 & 3 \\
\hline Insomnia & 4 & 1.7 \\
\hline Premenstrual tension & 3 & 1.3 \\
\hline Phobias & 2 & 0.8 \\
\hline Personality disorder & 2 & 0.8 \\
\hline Schizoaffective disorder & 2 & 0.8 \\
\hline Panic disorder & 1 & 0.4 \\
\hline Post-traumatic stress disorder & 1 & 0.4 \\
\hline Adjustment disorder & 1 & 0.4 \\
\hline Atypical grief & 1 & 0.4 \\
\hline Hypochondriasis & 1 & 0.4 \\
\hline Mood swings & 1 & 0.4 \\
\hline Schizophrenia & 1 & 0.4 \\
\hline
\end{tabular}

a. Total number of treatment episodes in the study sample $n=241$.

\section{Medication used}

Selective serotonin reuptake inhibitors (SSRIs) were found to be the most commonly used antidepressants (77\%) (Table 2). Mirtazapine and trazodone were used in $8.3 \%$ and $5.8 \%$ of the total treatment episodes respectively; tricyclics were noted to be used in only $5 \%$ of treatment episodes; serotonin noradrenaline reuptake inhibitors (venlafaxine and duloxetine) were used in $4 \%$ of episodes. Among the SSRIs, citalopram stood out unchallenged as the most favoured antidepressant $(43.2 \%$ of the episodes and $56 \%$ of the SSRIs use). No monoamine oxidase inhibitors had been used.

Co-prescription occurred with the majority of the treatment episodes with antidepressants - only 52 treatment episodes (21.5\%) were not associated with co-prescription. Antipsychotics (36\%), antiepileptic drugs (22.8\%), benzodiazepines (14.5\%), mood stabilisers (8.3\%) and proton pump inhibitors $(7.5 \%)$ were noted.

\section{Measuring treatment outcome}

The outcome of the antidepressant use was noted in terms of clinical improvement, retention or discontinuation and side-effects at follow-up at 6 weeks, 6 months and 12 months (Fig. 1). At 6 weeks, 119 treatment episodes (49.3\%) had a positive outcome, with significant improvement in 115 cases and with the patient being described as back to normal' in four cases. At 6 months, 116 episodes (48.1\%) had shown clinical improvement, 109 significant improvement and 7 back to normal. At 12 months, 117 episodes (48.5\%) had positive outcomes, with significant improvement in 97 and back to normal in 20 cases. Retention rates were 218 (90.4\%), $185(76.7 \%)$ and $159(65.9 \%)$ at 6 weeks, 6 months and 12 months respectively. There was a trend towards a gradual increase in discontinuation of antidepressants over the 1-year follow-up.

Table 2 Types of antidepressants used in the study sample and average dosage

\begin{tabular}{lccc} 
Antidepressant & Frequency & Per cent & $\begin{array}{c}\text { Average dose, mg } \\
\text { (range) }\end{array}$ \\
\hline Citalopram & 104 & 43.2 & $18.7(8-40)$ \\
\hline Fluoxetine & 43 & 17.8 & $20.6(10-60)$ \\
\hline Escitalopram & 21 & 8.7 & $11.10(5-20)$ \\
\hline Mirtazapine & 20 & 8.3 & $24.20(15-45)$ \\
\hline Trazodone & 14 & 5.8 & $122.8(50-400)$ \\
\hline Paroxetine & 9 & 3.7 & $22.5(10-40)$ \\
\hline Venlafaxine & 8 & 3.3 & $99.2(75-150)$ \\
\hline Sertraline & 7 & 2.9 & $52.5(25-100)$ \\
\hline Dothiepin & 7 & 2.9 & $140.5(25-175)$ \\
\hline Clomipramine & 2 & 0.8 & $69(10-75)$ \\
\hline Duloxetine & 2 & 0.8 & $30(30-30)$ \\
\hline Fluvoxamine & 1 & 0.4 & $37.5(25-50)$ \\
\hline Imipramine & 1 & 0.4 & 25 \\
\hline Amitriptyline & 1 & 0.4 & 30 \\
\hline Nortriptyline & 1 & 0.4 & 10 \\
\hline
\end{tabular}




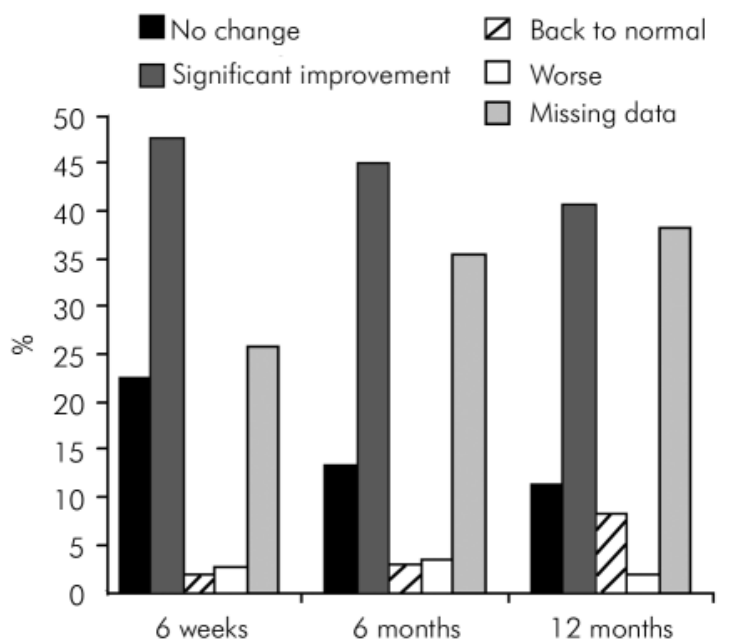

Fig 1 Clinical improvement at 6 weeks, 6 months and 12 months follow-up.

Out of 59 episodes of discontinuation noted, 20 (33.9\%) were due to switch to another antidepressant, 14 (23.7\%) due to non-response, $12(20.3 \%)$ due to side-effects and 3 (5.1\%) due to non-adherence. No reason had been recorded for 10 episodes (16.9\%) of discontinuation.

Only 29 episodes of side-effects had been recorded over the 1-year follow-up.

\section{Antidepressant side-effects recorded in the sample}

The use of SSRIs was associated with most of the adverse effects noted, reflecting the more prevalent use of these compounds. Four episodes each of sedation, stimulation, weight gain and gastrointestinal symptoms had been recorded, as well as three episodes of increased seizure activity and two episodes of increased anxiety. Citalopram, the most commonly used antidepressant, was associated with 15 episodes of side-effects viz. three episodes of sedation, two episodes of nausea and vomiting, two episodes each of hypomania and increased seizure, and one episode each of nausea, stimulation, hypotension, weight gain, increased anxiety and urinary symptoms. Of other antidepressants used, fluoxetine was responsible for two episodes of stimulation and one episode of increased anxiety; paroxetine was associated with one episode each of stimulation and sweating; dothiepin was associated with an episode of dry mouth and tremors; mirtazapine caused weight gain and sedation in one episode each; and trazodone was associated with weight gain on one occasion.

\section{Discussion}

\section{Study strengths and limitations}

The difficulty in carrying out, and an extreme lack of, randomised controlled trials (RCTs) in individuals with intellectual disability is well recognised. Apart from ethical and consent issues, common confounding factors such as polypharmacy and presence of physical conditions such as epilepsy make RCTs expensive, time consuming and often challenging in terms of recruitment. ${ }^{10}$
Data from alternative sources are therefore needed. Our study has the strength of being naturalistic and describing current clinical practice. However, because of the nature of the study - retrospective case-note analysis - it has limitations. As expected, the biggest problem was found to be related to the record keeping and missing data.

\section{Study findings}

The results reveal that antidepressants were commonly used in individuals with intellectual disability, both adults and elderly (14 service users $(6.3 \%)$ were aged above 65 years) for a range of conditions. Comorbidity was common, with the prevalence of epilepsy in our study group similar to that in individuals with intellectual disability in general, ${ }^{11}$ but the prevalence of challenging behaviour was found to be higher in the study sample. ${ }^{12}$

The majority of those receiving antidepressants had mild to moderate intellectual disability, which begs the question whether we are missing depression and anxiety disorders in service users with severe disability.

The recorded clinical outcome was good and even if the missing data were to be considered as treatment failures, nearly half the sample improved while on antidepressant treatment over the 1-year follow-up period. Of course, the study could not say whether this was drug effect or natural history of the disease. Furthermore, we lacked information on important comorbidity such as the presence of autismspectrum disorder. Information on differential prescribing and/or outcome in this group would have been valuable and merits future research.

Recording of side-effects seemed inconsistent and clinicians should consider more structured recording as we are likely to be missing these important data. Nonetheless, it was found that the majority of the service users received low average dosage of antidepressants. This may suggest that clinicians are cautious about the dosage and may in particular be concerned about potential side-effects.

The study shows that antidepressants are commonly prescribed in adults with intellectual disability. Approximately half of our sample did well in terms of clinical improvement. However, the lack of RCT data in individuals with intellectual disability and mental health problems is a concern.

\section{Acknowledgement}

We thank the secretarial and consultant staff for their kind support.

\section{About the authors}

Prem R. Rai is Locum Consultant in Learning Disability Psychiatry, Aneurin Bevan Health Board, Cwmbran, Torfaen, and Mike Kerr is Professor of Learning Disability Psychiatry, Welsh Centre for Learning Disability, Cardiff.

\section{References}

1 Cooper SA, Smiley E, Morrison J, Williamson A, Allan L. Mental illhealth in adults with intellectual disabilities: prevalence and associated factors. Br J Psychiatry 2007; 190: 27-35. 
2 Singleton N, Bumpstead R, O'Brien M, Lee A, Meltzer H. Psychiatric Morbidity among Adults Living in Private Households: Summary Report. Office for National Statistics, 2000.

3 Howland RH. Fluoxetine treatment of depression in mentally retarded adults. J Nerv Ment Dis 1992; 180: 202-5.

4 Langee HR, Conlon M. Predictors of response to antidepressan medications. Am J Ment Retard 1992; 97: 65-70.

5 Verhoeven WMA, Veendrik-Meekes MJ, Jacobs GAJ, van den Berg YWMM, Tuinier S. Citalopram in mentally retarded patients with depression: a long-term clinical investigation. Eur Psychiatry 2001; 16: $104-8$

6 Bhaumik $S$, Branford $D$, Naik $B I$, Biswas $A B$. A retrospective audit of selective serotonin re-uptake inhibitors (fluoxetine and paroxetine) for the treatment of depressive episodes in adults with learning disability. Br J Dev Disabil 2000; 46: 131-9.
7 Branford D, Bhaumik S, Naik B. Selective serotonin receptor inhibitors treatment of perseverative and maladaptive behaviours of people with intellectual disability. J Intellec Disabil Res 1998; 42: 301-6.

8 Singh AN, Kleynhans D, Barton G. Selective serotonin reuptake inhibitors in the treatment of self-injurious behaviour in adults with mental retardation. Hum Psychopharmacol Clin Exp 1998; 13: 267-70.

9 Lunsky Y, Palucka A. Depression and intellectual disability:a review of current research. Curr Opin Psychiatry 2004; 17: 359-63.

10 Lennox N, Taylor M, Rey-Conde T, Bain C, Purdie DM, Boyle F. Beating the barriers: recruitment of people with intellectual disability to participate in research. J Intellect Disabil Res 2005; 49: 296-305.

11 Morgan $\mathrm{CL}$, Baxter $\mathrm{H}$, Kerr MP. Prevalence of epilepsy and associated health service utilisation and mortality among patients with intellectual disability. Am J Ment Retard 2003; 108: 293-300.

12 Emerson E, Kiernan E, Alborz A, Reeves D, Mason H, Swarbrick R, et al. The prevalence of challenging behaviours: a total population study. Res Dev Disabil 2001; 22: 77-93.

\title{
Antipsychotic prescribing practice among child psychiatrists and community paediatricians
}

\author{
John Otasowie, ${ }^{1}$ Rachel Duffy, ${ }^{2}$ Jenny Freeman, ${ }^{3}$ Chris Hollis ${ }^{4}$
}

The Psychiatrist (2010), 34, 126-129, doi: 10.1192/pb.bp.108.024000

${ }^{1}$ Worcestershire Specialist Children's Services; ${ }^{2}$ Alder Hay Children's NHS Foundation Trust; ${ }^{3}$ University of Sheffield; ${ }^{4}$ University of Nottingham Correspondence to John Otasowie (john.otasowie@worcspct.nhs.uk)
Aims and method All child and adolescent psychiatrists and community paediatricians in the former Trent Region were surveyed about their antipsychotic prescribing practice during 1 year, including monitoring, and whether they would like consensus guidelines on prescribing and monitoring of antipsychotics in children and adolescents.

Results The majority (88\%) of child psychiatrists and 33\% of paediatricians had prescribed atypical antipsychotics, most commonly risperidone. Only two psychiatrists had prescribed a typical antipsychotic and no paediatrician had done so. Challenging behaviour in developmental disorders was the most common indication for atypicals. Both child psychiatrists and paediatricians prescribed atypicals for nonpsychotic developmental disorders, whereas prescribing for psychosis occurred almost exclusively among psychiatrists. Height, weight and blood pressure were routinely monitored, but waist circumference was rarely measured and there was wide variation in the monitoring of other parameters such as blood glucose, prolactin and extrapyramidal side-effects. Three-quarters of the participants felt there was a need for guidance on prescribing and monitoring atypical antipsychotic therapy.

Clinical implications The greater prescription of antipsychotics by child and adolescent psychiatrists may reflect differences in case-load and training. Routine monitoring of adverse effects is inconsistent among prescribers. The survey highlights the need for training and guidance on prescribing and monitoring of atypical antipsychotic use in children and adolescents.

Declaration of interest None.
Atypical antipsychotic medication is increasingly prescribed 'off label' to children and adolescents. Reasons for changing prescribing practice are various and may include an increased evidence base of efficacy, concerns about the adverse effect profile of typical antipsychotics in young people, better training in paediatric psychopharmacology, greater drug availability and promotion, and possibly also pressure on clinicians to act promptly and unavailability of non-pharmacological interventions. ${ }^{1,2}$ In children there is evidence that most atypical antipsychotics are prescribed 